\title{
Efficiency of Go/No-Go Task Performance Implemented in the Left Hemisphere
}

\author{
Satoshi Hirose, ${ }^{1}$ Junichi Chikazoe, ${ }^{1}$ Takamitsu Watanabe, ${ }^{1}$ Koji Jimura, ${ }^{1}$ Akira Kunimatsu, ${ }^{2}$ Osamu Abe, ${ }^{2}$ \\ Kuni Ohtomo, ${ }^{2}$ Yasushi Miyashita, ${ }^{1}$ and Seiki Konishi ${ }^{1}$ \\ Departments of ${ }^{1}$ Physiology and ${ }^{2}$ Radiology, The University of Tokyo School of Medicine, 7-3-1 Hongo, Bunkyo-ku, Tokyo 113, Japan
}

It is well known that the efficiency of response inhibition differs from person to person, but the neural mechanism that implements the efficiency is less understood. In the present fMRI study, we devised an index to evaluate the efficiency of response inhibition in the go/ no-go task, and investigated the neural correlates of the efficiency of response inhibition. The human subjects who perform the go/no-go task with a shorter reaction time in go trials (Go-RT) and with a higher percentage of correct no-go trials (Nogo-PC) are thought to have the ability to conduct response inhibition more efficiently. To quantify the efficiency, we defined an efficiency index as the difference in the Nogo-PC between each subject and an ordinarily efficient subject, under the same Go-RT. An across-subject correlation analysis revealed that the brain activity in multiple regions in the left frontal and parietal cortex positively correlated with the efficiency index. Moreover, a test of hemispheric asymmetry with regard to the across-subject correlation revealed left-hemispheric dominance. The significant correlation in the left frontal and parietal regions complements the results of previous studies that used the stop-signal reaction time (SSRT), a well known index to evaluate the efficiency of response inhibition used in the stop-signal task. Our results also indicate that, although it is well known that the neural substrates for response inhibition common in a subject group exist dominantly in the right hemisphere, the neural substrates for efficiency exist dominantly in the left hemisphere.

\section{Introduction}

Although response inhibition has been well studied with regard to the brain activation common to a subject group by contrasting, for example, the no-go versus go trials (Garavan et al., 1999; Konishi et al., 1999; Liddle et al., 2001; Menon et al., 2001; Bunge et al., 2002; Durston et al., 2002; Hester et al., 2004; Kelly et al., 2004; Matsubara et al., 2004; Aron and Poldrack, 2006; Li et al., 2006; Leung and Cai, 2007; Zheng et al., 2008; Chikazoe et al., 2009a,b; Mander et al., 2010; van Gaal et al., 2010; Jahfari et al., 2011), the neural correlates of the efficiency of response inhibition is relatively less understood. A stop-signal reaction time (SSRT) is a well known index defined in a stop-signal task (Logan and Cowan, 1984; Rubia et al., 2001; Aron et al., 2003; Li et al., 2006; Chikazoe et al., 2009b) that is used to evaluate the efficiency of response inhibition. According to a horse-race model (Logan and Cowan, 1984), which accounts for response inhibition as a race between a "go process" and a "stop process," SSRT is the time it takes for a subject to

Received Feb. 4, 2012; revised April 25, 2012; accepted May 4, 2012.

Author contributions:S.H. and S.K. designed research;S.H., J.C., A.K., O.A., K.O., and S.K. performed research;S.H. analyzed data; S.H., J.C., T.W., K.J., Y.M., and S.K. wrote the paper.

S.H. is supported by a Japan Society for the Promotion of Science Fellowship (20-6285) for Young Scientists. This work was supported by a Grant-in-Aid for Specially Promoted Research (19002010) to Y.M.; a Grant-in-Aid for Scientific Research B (22300134) to S.K.; Global Center of Excellence Program "Integrative Life Science Based on the Study of Biosignaling Mechanisms" (12601-A03) to Y.M. and J.C. from the Ministry of Education, Culture, Sports, Science and Technology, Japan; and by the Takeda Science Foundation.

Correspondence should be addressed to either of the following Dr. Satoshi Hirose, Department of Physiology, The University of Tokyo School of Medicine, 7-3-1 Hongo, Bunkyo-ku, Tokyo 113, Japan, E-mail: sahirose@m.u-tokyo. ac.jp, or Dr. Seiki Konishi, Department of Physiology, The University of Tokyo School of Medicine, 7-3-1 Hongo, Bunkyo-ku, Tokyo 113, Japan. E-mail: konishi@m.u-tokyo.ac.jp.

DOI:10.1523/JNEUROSCI.0540-12.2012

Copyright $\odot 2012$ the authors $\quad 0270-6474 / 12 / 329059-07 \$ 15.00 / 0$ suppress a response after a stop signal. Subjects are thought to conduct response inhibition more efficiently when they perform the stop-signal task with a shorter SSRT. It has been revealed that the brain activity of several regions in the frontal cortex correlates negatively with SSRT (i.e., the shorter the SSRT, the greater the brain activity in the regions) (Li et al., 2006, 2008).

The stop-signal task presents a go signal at the beginning of a stop trial, followed by a stop signal after a short delay (stop-signal delay, SSD). SSD is modulated on a trial-by-trial basis to keep accuracy in stop trials constant (Band et al., 2003), such that SSRT can be calculated based on SSD and a distribution of reaction time of go trials (Logan and Cowan, 1984). In contrast to the sophisticated structure of the stop-signal task, a go/no-go task (Chikazoe et al., 2009a) is simpler and does not have SSD. Using the go/no-go task we devised an alternative way to evaluate the efficiency of response inhibition by elaborating on the analysis of acquired behavioral data, and determined the neural correlates of the efficiency of response inhibition. In the go/no-go task, the subjects with higher efficiency ought to have a shorter reaction time in go trials (Go-RT) and a higher percentage of correct performance in no-go trials (Nogo-PC). The efficiency index was defined as the difference in the Nogo-PC between a subject and an ordinarily efficient subject under the same Go-RT. To investigate the neural correlates of the efficiency of response inhibition, we then calculated the correlations between the brain activity and the efficiency index. Further, we compared the results of the present study with those reported in previous studies that used SSRT, and examined the consistency of neural correlates of response inhibition, as defined by SSRT and the efficiency index of the present study. 

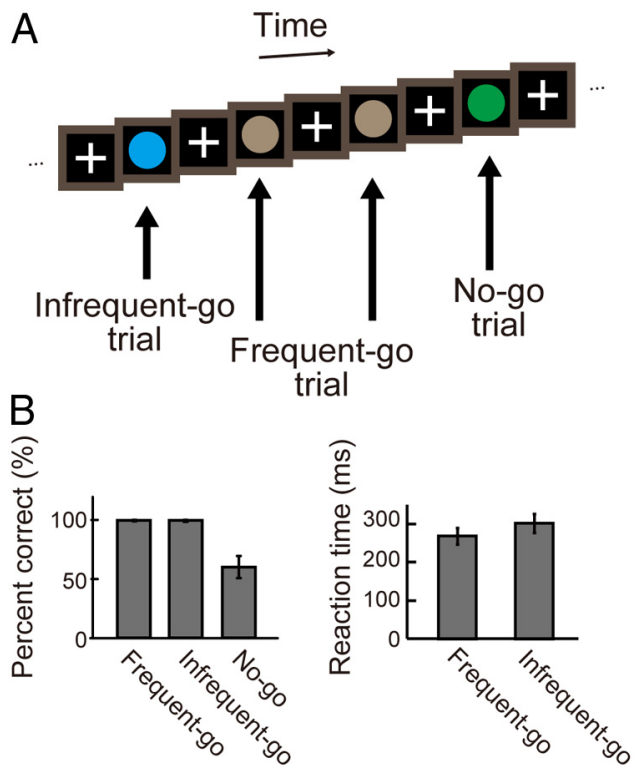

Figure 1. A, The go/no-go task used in the present study. The task contained three types of trials: frequent-go, infrequent-go, and no-go trials. The no-go trials are contrasted with the infrequent-go trials to examine response inhibition. $\boldsymbol{B}$, The behavioral results. Left, the percentage of correct trials in the frequent- $\mathrm{g} 0$, infrequent- $\mathrm{g} 0$, and no-go trials. Right, the reaction times in the frequent-go and infrequent-go trials. Error bars indicate SD.

\section{Materials and Methods}

Subjects. Informed consent was obtained from 59 healthy right-handed subjects ( 29 males; 30 females, age: $20-30$ years). They were scanned using experimental procedures approved by the institutional review board of the University of Tokyo School of Medicine.

MRI procedures. The experiments were conducted using a $3 \mathrm{~T}$ fMRI system. Scout images were first collected to align the field of view centered on the subject's brain. T1-weighted images were obtained for anatomical reference (76 slices $\times 2 \mathrm{~mm}$ slices; in-plane resolution $=1 \times 1$ $\mathrm{mm})$. For functional imaging, a gradient echo echo-planar sequence was used $(\mathrm{TR}=3000 \mathrm{~ms} ; \mathrm{TE}=35 \mathrm{~ms}$; flip angle $=90$ degrees; $40 \times 4 \mathrm{~mm}$ slices; in-plane resolution of $4 \times 4 \mathrm{~mm}$ ). Each run contained 60 volume images, and the first four functional images in each run were excluded from the analysis to take into account the equilibrium of longitudinal magnetization.

Task. The go/no-go task used in the present study consisted of three types of trial: frequent-go, infrequent-go, and no-go trials (Chikazoe et al., 2009a) (Fig. 1A). In the frequent-go and infrequent-go trials, the subjects were required to press a button with a right thumb, and in the no-go trial, the subjects were required not to press the button, withholding the prepotent response tendency. Each trial consisted of presentation of a colored circle for $400 \mathrm{~ms}$ and presentation of a fixation cross for 400 ms. There was no other interval between the trials. A time window to record a response was $800 \mathrm{~ms}$, and the subjects were instructed to press a button by the end of the circle presentation in the go trials. To exclude potentially premature responses, go trials with an RT of $<150 \mathrm{~ms}$ were excluded from analysis. The color of the circle indicated the type of trial: gray indicated the frequent-go trial, whereas green and blue indicated the infrequent-go and no-go trial, respectively. The relationship between color (green/blue) and trial type (infrequent-go/ no-go) was counterbalanced across subjects. The percentage of no-go, infrequent-go and frequent-go trials was $12.5 \%, 12.5 \%$, and $75.0 \%$, respectively, and these types of trials were presented pseudo-randomly. One run consisted of 192 trials, and filler frequent-go trials were also presented at the beginning and end of each run ( 15 trials at the beginning and 10 trials at the end). Four to 12 runs were collected for each of the subjects.

Data analysis. Data were analyzed using SPM8 software (http://www. fil.ion.ucl.ac.uk/spm/). Functional images were realigned, slice timing corrected, normalized to the Montreal Neurological Institute template

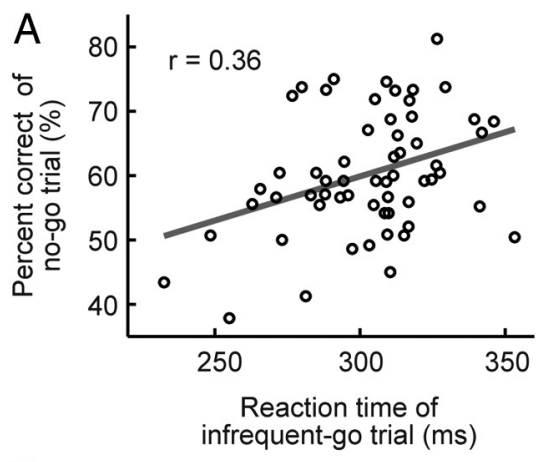

B

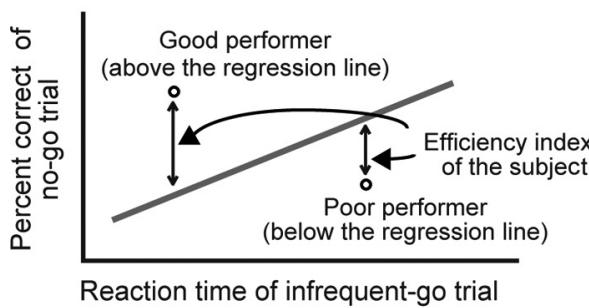

C

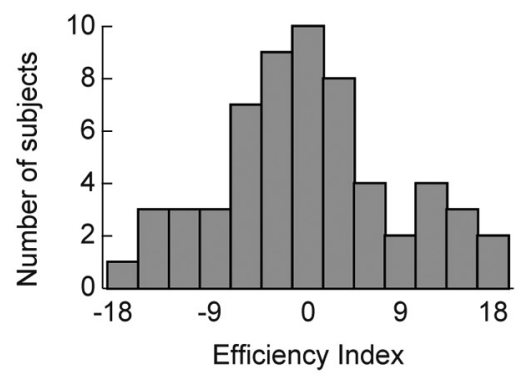

Figure 2. $\quad \boldsymbol{A}$, The distribution of the reaction time in the infrequent-go trials and the percentage accuracy in the no-go trials. One dot represents one subject. The gray line indicates the regression line. $\boldsymbol{B}$, The diagram to explain the efficiency index invented in the present study. The percentage accuracy at a given reaction time in the infrequent-go trials in one subject, relative to that in a standard performer indicated by the gray line, was defined as the efficiency index. $\boldsymbol{C}$, The distribution of the efficiency index.

with interpolation to a $2 \times 2 \times 2 \mathrm{~mm}$ space, and spatially smoothed (full width half maximum $=8 \mathrm{~mm}$ ). Then event timing was coded into a general linear model (Worsley and Friston, 1995). Transient events at the time of the infrequent-go trials, the correct no-go trials, and the incorrect no-go trials were modeled as events using the canonical function in SPM8, together with run-specific regressors as effects of no interest. The frequent-go trials were used as a baseline, and were not coded as events. The brain activation associated with response inhibition was calculated based on the contrast of no-go versus infrequent-go trials. Group analyses were conducted using a random-effects model. Significant activations were detected using a threshold of $p<0.05$ corrected by the false discovery rate (FDR; Genovese et al., 2002).

To evaluate the efficiency of response inhibition in the go/no-go task, the efficiency index was calculated. A subject who performs the task with a shorter reaction time in infrequent-go trials (Go-RT) and a higher percentage of correct performance in no-go trials (Nogo-PC) ought to be regarded as a more efficient performer. The efficiency index was defined as the difference in the Nogo-PC between a subject and an ordinarily efficient subject under the same Go-RT (Fig. 2 B). The performance of the ordinarily efficient subject was represented by a regression line between the Go-RT and the Nogo-PC from all of the subjects (Fig. 2 B).

The efficiency index was then used to investigate the neural correlates of the efficiency of response inhibition in an across-subject correlation analysis. We calculated voxel-by-voxel correlations across subjects between the efficiency index and the magnitude of the brain activation associated with response inhibition. Significant correlations were de- 
tected using a threshold of $p<0.05$ corrected by the FDR within a mask of the gray-matter in the cerebral cortex derived from SPM8. Further, to test the consistency between the results of the present study and those of the previous studies that used SSRT, a small volume correction (SVC) was applied using the coordinates reported in the previous study (Li et al., 2006) with a threshold of $p<0.05$ corrected by the family-wise error correction.

We then tested the hemispheric asymmetry with regard to the acrosssubject correlations. The voxelwise $t$-values for correlations between the brain activation and the efficiency index were converted to $z$-score. Next, to compare the correlations between the right and the left hemisphere in an unbiased way, the $z$-map was flipped along the midline of the brain, and the original $z$-map and the flipped $z$-map were averaged and multiplied by a square root of 2 . The local maxima of the resultant $z$-map above the threshold of $p<0.001$ were set as the center of bilateral pairs of ROIs (radius: $12 \mathrm{~mm}$ ). We then compared $\beta$-values for the correlations in the right hemisphere with those in the left hemisphere in each of the paired ROIs.

To test the hemispheric asymmetry without the assumption that the right and left hemispheres are structurally homologous, we conducted two additional analyses by determining the ROIs contralateral to the correlation peaks as follows. In the first analysis, a resting-state fMRI dataset from our previous study (Kimura et al., 2010) was used. The peaks in the map of correlation between the brain activity and the efficiency index above the threshold of $p<0.001$ were set as the center of ROIs, and the largest resting-state connectivity peaks in the contralateral hemisphere within $8 \mathrm{~mm}$ of the mirrored peaks were determined as the center of contralateral ROIs. In the second analysis, ROIs were determined using only the map of correlation between the brain activity and the efficiency index. The peaks in the correlation map above the threshold of $p<0.001$ were set as the center of ROIs, and the largest correlation peaks in the contralateral hemisphere within $8 \mathrm{~mm}$ of the mirrored peaks were determined as the center of contralateral ROIs. In some cases, contralateral ROIs were located closely to one another. To assure the independent samples of the ROIs in such cases, in the above two analyses, data of the bilateral ROIs within $12 \mathrm{~mm}$ were averaged into one sample.

Additional experiment. To confirm that the efficiency index in the present study truly reflects efficiency of response inhibition, we conducted a behavioral experiment where each subject performed both the stop-signal task and the go/no-go task. In the stop-signal task, of the 59 subjects recruited in the main experiment, 29 of them were also recruited to perform the stop-signal task. In the go/no-go task, behavioral data of the 29 subjects were taken from the main experiment. Nine subjects were separately recruited to perform both the two tasks. Therefore, behavioral data from 38 subjects were compared between the two tasks.

The stop-signal task was similar to that used in our previous study (Chikazoe et al., 2009b) except that the certain-go trials were not included. Thus, the stop-signal task in this experiment consisted of stop trials $(25 \%)$ and go trials (75\%), where a circle of one color (green/blue) was presented as a go signal and a circle of the other color (blue/green) was presented as a stop signal. In the go trials, the go signal was presented, and subjects were required to press a button using the right thumb. In the stop trials, the go signal was first presented and, after a short delay (SSD), the stop signal was presented, and subjects were required to withhold the response. One trial lasted for $800 \mathrm{~ms}$, followed by an intertrial interval of $1700 \mathrm{~ms}$. A time window to record a response was $800 \mathrm{~ms}$. To exclude potentially premature responses, go trials with an RT of $<150 \mathrm{~ms}$ were excluded from analysis. The relationship between colors (green and blue) and trial type (go and stop/no-go) was consistent across the two tasks. Twelve sessions of 64 trials were administered for each subject. The SSD started at $200 \mathrm{~ms}$ and varied from one stop trial to the next by $33 \mathrm{~ms}$ based on to a tracking procedure (Band et al., 2003), and the percentage correct in the stop trials was 50\%. The SSRT was estimated for each subject using the integration method devised by Logan and Cowan (1984).

\section{Results}

\section{Behavioral results}

In the go/no-go task, mean percentages of correct performance $($ mean \pm SEM) were $99.7 \pm 0.1 \%, 99.6 \pm 0.1 \%$ and $60.3 \pm 1.2 \%$ in the frequent-go, infrequent-go, and no-go trials, respectively (Fig. $1 B$ ). The difference in the percentage of correct performance between the frequent-go and the no-go trials was significant $\left(t_{(58)}=32.74, p<0.001\right)$, and the difference between the infrequent-go and the no-go trials was also significant $\left(t_{(58)}=\right.$ 32.69, $p<0.001$ ). Mean reaction times (mean \pm SEM) were $268.2 \pm 2.8 \mathrm{~ms}$ and $302.8 \pm 3.2 \mathrm{~ms}$ in the frequent-go and the infrequent-go trials, respectively (Fig. $1 B$ ), and the difference between the frequent-go and the infrequent-go trials was significant $\left(t_{(58)}=17.5, p<0.001\right)$. The reaction time difference between the infrequent-go and frequent-go trials indicates that the infrequent-go trials contained sufficient amount of processes associated with processing of infrequent stimuli (Chikazoe et al., 2009a).

To calculate the efficiency index (see Materials and Methods, Data analysis), we conducted the linear regression analysis between the reaction time in the infrequent-go trials (Go-RT) and the percentage of correct performance in the no-go trials (NogoPC) (Fig. 2A). The Go-RT and the Nogo-PC was significantly correlated $\left(r=0.36, t_{(57)}=2.9, p<0.01\right)$. The regression line between the Go-RT and the Nogo-PC was used as a performance of an ordinarily efficient subject to calculate the efficiency index (Fig. $2 \mathrm{~B}$ ). A well balanced distribution of the efficiency index was obtained (Fig. 2C). The distribution was not significantly different from a normal one as tested by KolmogorovSmirnov test $(p=0.87$ ), and the SD of the distribution of the efficiency index was 8.7 .

In the stop-signal task conducted in an additional experiment, mean percentages of correct performance (mean \pm SEM) were $98.7 \pm 0.2 \%$, and $49.9 \pm 0.2 \%$ in the go and stop trials, respectively. A mean reaction time (mean \pm SEM) in the go trials was $523.2 \pm 10.9 \mathrm{~ms}$. A mean stop signal delay (mean \pm SEM) in the stop trials was $318.2 \pm 13.5 \mathrm{~ms}$. A mean SSRT (mean \pm SEM) was $202.4 \pm 5.4 \mathrm{~ms}$.

\section{fMRI results}

The brain activation associated with response inhibition was calculated based on the contrast of no-go versus infrequent-go trials (Chikazoe et al., 2009a). The magnitude of the brain activation in each subject was entered into a second-level group analysis using a random effects model. As shown in Figure 3, the prominent activations were found in multiple regions primarily in the right hemisphere, including the dorsolateral prefrontal cortex, the insula, the inferior frontal gyrus (IFG), the presupplementary motor area (pre-SMA), the temporoparietal junction (TPJ), the intraparietal sulcus (IPS), consistent with the results of the previous studies that used the go/no-go task or the stop-signal task (Garavan et al., 1999; Konishi et al., 1999; de Zubicaray et al., 2000; Liddle et al., 2001; Menon et al., 2001; Rubia et al., 2001; Bunge et al., 2002; Durston et al., 2002; Hester et al., 2004; Kelly et al., 2004; Matsubara et al., 2004; Aron and Poldrack, 2006; Li et al., 2006; Aron et al., 2007; Brass and Haggard, 2007: Leung and Cai, 2007; Forstmann et al., 2008; Xue et al., 2008; Zheng et al., 2008; Chikazoe et al., 2009a,b; Mander et al., 2010; van Gaal et al., 2010; Jahfari et al., 2011).

To investigate the neural correlates of the efficiency of response inhibition, we conducted a correlation analysis across subjects. The significant positive correlations between the brain activation (no-go vs infrequent-go trials) and the efficiency index were detected in the left IFG and the left TPJ (Fig. 4 and Table 1). Further, the significant correlations were detected in the left superior frontal gyrus (SFG) and the left precentral gyrus/middle frontal gyrus (PreCG/MFG) using a SVC based on the coordi- 
nates reported in the previous study that used SSRT as an index of the efficiency of response inhibition (Li et al., 2006) (Table $1)$. On the other hand, no significant negative correlation was detected, even when the threshold was lowered to $p<0.001$ (uncorrected), which suggests that there were no neural correlates of the inefficiency of response inhibition.

The four significant correlations described above were all in the left hemisphere (Table 1). We then tested the hemispheric asymmetry with regard to the correlation between the brain activation and the efficiency index. The $t$-values for the correlations between the brain activation and the efficiency index were converted to $z$-score, and the flipped and original $z$-maps were averaged and multiplied by a square root of 2 (see Materials and Methods, Data analysis). Eighteen bilateral pairs of ROIs were generated as a result. We then compared the $\beta$-values for correlations in the right hemisphere with those in the left hemisphere in each of the 18 pairs of ROIs. Typical examples of the ROIs are shown in Figure $5 A$. Note that there was no error bar for the $\beta$-values for the correlations in each ROI, because the correlation can be calculated only after the data from all the subjects are considered. The comparison of the $\beta$-values between the right and the left hemisphere was conducted based on the variance of the $\beta$-values across the 18 ROIs. The comparison revealed significant left-hemispheric dominance among these ROIs $\left(t_{(17)}=3.6, p<0.005\right)$.

We further analyzed the hemispheric asymmetry without the assumption that the right and left hemispheres are structurally homologous (see Materials and Methods, Data analysis). In the first analysis using the resting-state fMRI (Fig. 5B), 15 bilateral pairs of ROIs were generated, and the left-hemispheric dominance was significant $\left(t_{(14)}=5.8, p<0.001\right)$. In the second analysis using the correlation data alone (Fig. 5C), 12 bilateral pairs of ROIs were generated, and the left-hemispheric dominance was significant $\left(t_{(11)}=4.2, p<0.005\right)$. These results confirmed the left-hemispheric dominance independently of the homologous structure assumption.

Given the left hemisphere-dominant correlation and the well known right-hemispheric brain activation associated with response inhibition, we next examined whether the four significant correlations in the left hemisphere had contralateral counterpart brain activation in the right hemisphere. The voxel in the right hemisphere contralateral to the correlation peak in the left hemisphere was set as the center of a ROI, and the brain activity in each of the four ROIs in the right hemisphere was examined. The results are listed in Table 1 . Interestingly, the brain activation in the counterpart right IFG was not significant $\left(t_{(58)}=0.1, p>\right.$ $0.05)$, whereas the brain activity in the counterpart right TPJ was significant $\left(t_{(58)}=2.2, p<0.05\right)$ (Table 1$)$.

\section{Correlation of behavioral indices of two inhibition-related tasks}

To confirm that the efficiency index in the present study truly reflects efficiency of response inhibition, an additional experi- ment was conducted where subjects performed both the stopsignal task and the go/no-go task (see Materials and Methods, Additional experiment). As shown in Figure 6, the SSRT (a smaller SSRT indicates greater efficiency) in the stop-signal task and the efficiency index (a larger index indicates greater efficiency) in the go/no-go task showed a significant anti-correlation $\left(r=-0.38, t_{(36)}=-2.5, p<0.05\right)$. These results suggest that the efficiency index reflects the efficiency of response inhibition, if we assume that the SSRT reflects the efficiency of response inhibition. However, because the correlation is only marginally significant, it is not the case that the two indices measure the same set of cognitive components.

\section{Discussion}

In the present study we invented the efficiency index, which is aimed at evaluating the efficiency of response inhibition in the go/no-go task. Significant correlations between the brain activation and the efficiency index across subjects were detected in the IFG, the SFG, the PreCG/MFG, and the TPJ in the left hemisphere. A test of hemispheric asymmetry in the correlation further revealed left-hemispheric dominance. These results indicate that there exist neural substrates of response inhibition in the left hemisphere that are modulated by the efficiency, whereas in the right hemisphere there exist the well known neural substrates of response inhibition (Konishi et al., 1998; Garavan et al., 1999; Bunge et al., 2002; Horn et al., 2003; Maguire et al., 2003; Hester et al., 2004; Rubia et al., 2005; Vink et al., 2005; Li et al., 2006; Chikazoe et al., 2007; Morimoto et al., 2008; Nakata et al., 2008; Xue et al., 2008; Zheng et al., 2008; Cai and Leung, 2009, 2011a; Coxon et al., 2009; Roberts and Garavan, 2010; Zandbelt and Vink, 2010; Lenartowicz et al., 2011) and other inhibitory functions that are common to the subjects (Milham et al., 2002, 2003; Hazeltine et al., 2003; Brass et al., 2005; Chambers et al., 2006; 


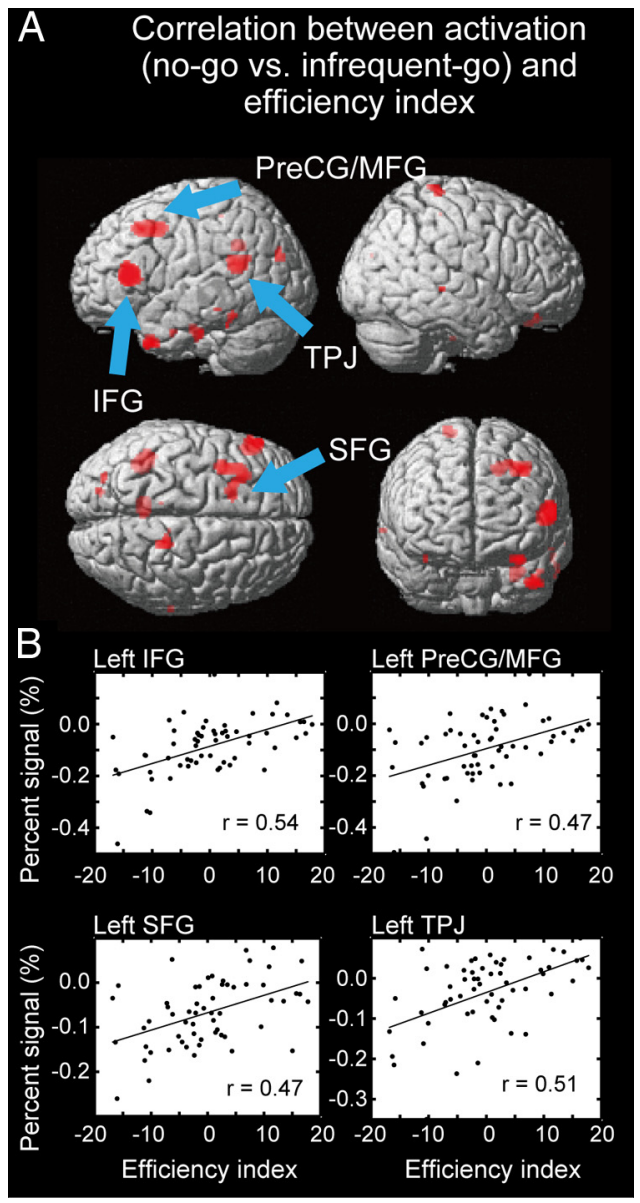

Figure 4. A, Statistical maps for correlation between the brain activation related to response inhibition and the efficiency index. Significant correlations were shown in red, overlaid on the surface of the three dimensional brain. $\boldsymbol{B}$, The scatter diagram between the percentage signals related to response inhibition and the efficiency index in the regions detected as significant. One dot represents one subject. The black line in each panel indicates a regression line.

Table 1. Significant correlations between the brain activations and the efficiency index across subjects

\begin{tabular}{lrllll}
\hline$X$ & $Y$ & $Z$ & $t$ (correlation) & $t$ (contralateral activation) & Area \\
\hline-54 & 30 & 12 & $4.9^{*}$ & 0.1 & IFG \\
-42 & -48 & 20 & $4.5^{*}$ & 2.2 & TPJ \\
-36 & 6 & 46 & $4.2^{* *}$ & 2.6 & PreCG/MFG \\
-18 & 14 & 46 & $4.0^{* *}$ & 1.4 & SFG \\
\hline
\end{tabular}

${ }^{*} p<0.05$, corrected by the FDR.

${ }^{* *} p<0.05$, corrected by the family-wise error (SVC).

Feredoes et al., 2006; Hodgson et al., 2007; Eagle et al., 2008; Verbruggen et al., 2010; Severens et al., 2012).

The left hemispheric dominance of correlation with efficiency is consistent with a neuropsychological study that showed that the left IFG is critical for response inhibition under a harder condition (Swick et al., 2008), and may imply that the left hemisphere plays a supplementary role in response inhibition when the right hemisphere is already fully engaged. Another possibility regarding the left/right asymmetry is that the right hemispheric processes may serve to orient attention in preparation to no-go trials (Chao et al., 2009; Duann et al., 2009; Hampshire et al., 2010). In particular, recent work of Zhang and Li (2012) using ICA of the stop signal task data showed that the right frontoparietal network implements attentional monitoring, whereas the left

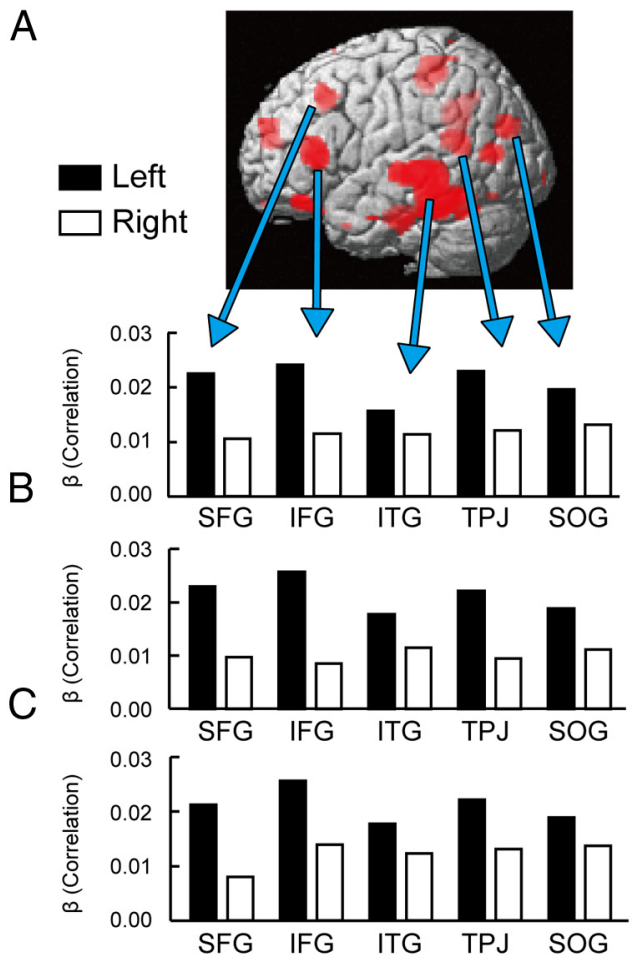

Figure 5. A, Five typical examples of regions showing hemispheric asymmetry in the magnitude of the correlations. Significant correlations after integrating bilateral correlations (see Materials and Methods, Data analysis) are shown in red on the surface of the three dimensional brain. ITG: inferior temporal gyrus, SOG: superior occipital gyrus. $\boldsymbol{B}$, The magnitude of the correlation in the above five regions as calculated by determining contralateral regions of interest based on the largest resting-state functional connectivity peaks within a small sphere around mirrored peaks. $C$, The magnitude of the correlation in the five regions as calculated by determining contralateral regions of interest based on the largest correlation peak within a small sphere around mirrored peaks.

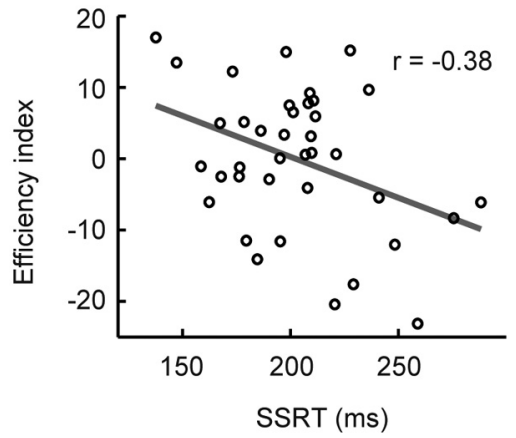

Figure 6. Correlation between SSRT in the stop-signal task and the efficiency index in the go/no-go task. Each dot represents one subject. The gray line indicates a regression line.

frontoparietal network implements response inhibition. Moreover, the attentional account of the right IFG activation is also supported by the observation that the right IFG was activated even during conditions where neither motor responses nor inhibition of the motor responses were required (Hampshire et al., 2010). These possibilities may not easily be reconciled, and further studies may elucidate clearer insights into the left/right asymmetry of hemispheric contribution to response inhibition.

The present study used the go/no-go task where a no-go cue indicates a less frequent event of stopping. One possibility is that the process of interpreting the cue is more difficult in the no-go trials than in go trials, which may introduce confounds in the 
contrast of no-go versus go trials. Although we cannot completely exclude the possibility, previous literatures have shown that the brain activity associated with response inhibition as investigated with the go/no-go task is largely similar, including the inferior frontal and medial frontal activations, to that as investigated with the stop-signal task (Rubia et al., 2001; Zheng et al., 2008; Chikazoe, 2010; Swick et al., 2011). Moreover, other task paradigms such as the stop-signal task may contain other types of confounds such as auditory processing associated with a stop signal. Thus, although response inhibition may depend on the task used for investigation, it would be difficult to conclude that any one particular task is better suited to investigate response inhibition.

The previous studies of response inhibition have used SSRT in the stop-signal task as an index for the efficiency of response inhibition (Logan and Cowan, 1984; Aron et al., 2003; Li et al., 2006; Chikazoe et al., 2009b, Cai et al., 2011b, Forstmann et al., 2012), and the efficiency index invented in the present study has both advantages and disadvantages over SSRT. The efficiency index in the present study has an advantage in that the index is based on a simpler assumption than SSRT. SSRT is calculated based on a horse-race model, and a sophisticated structure of the stop-signal task is necessary to calculate SSRT based on the horserace model. On the other hand, the efficiency index in the present study is not based on the horse-race model, and can be calculated using the go/no-go task, which is much simpler than the stopsignal task. Conversely, SSRT has an advantage over the efficiency index in the present study, in that SSRT for one subject can be calculated based on behavioral data of one subject. Behavioral data of a group population is necessary to calculate the efficiency index in the present study for one subject, and the index indicates a relative efficiency of response inhibition in that population. Despite these differences between the two indices, the correlation between the index and the brain activation exhibited consistency in the left SFG and PreCG/MFG (Li et al., 2006). The consistency indicates the validity of both SSRT and the efficiency index in the present study as an index of the efficiency of response inhibition, and also provides support for the validity of the horse-race model (Logan and Cowan, 1984; Verbruggen and Logan, 2008) to explain the neural mechanism of response inhibition.

The present study using the efficiency index further revealed significant correlations between the efficiency index and the brain activation in the left IFG and TPJ. Our previous study focusing on the precise localization of the IFG activation in the right hemisphere associated with response inhibition has revealed that the $Y$ coordinates of the right IFG activation ranges from 8 to 18 (Hirose et al., 2009). The significant correlation in the IFG in the left hemisphere $(Y=30)$ is located more anterior to the typical right IFG activation. Indeed, the brain activity in the counterpart right IFG region was not significant (Table 1). The apparent spatial discrepancy in these regions suggests the heterogeneity of the IFG where multiple neural substrates may contribute to response inhibition in different manners. On the contrary, the right TPJ, located in the contralateral part of the left TPJ that showed significant positive correlation, also showed significant brain activity, which indicates the bilateral TPJ activation in efficient performers. Although these results do not fully uncover the neural mechanisms of response inhibition in terms of the mechanisms that are common in the subjects or are modulated depending on the efficiency of the subjects, the present study provides a crucial distinction on their neural correlates that is exhibited as hemispheric asymmetry.

\section{References}

Aron AR, Poldrack RA (2006) Cortical and subcortical contributions to stop signal response inhibition: role of the subthalamic nucleus. J Neurosci 26:2424-2433.

Aron AR, Fletcher PC, Bullmore ET, Sahakian BJ, Robbins TW (2003) Stopsignal inhibition disrupted by damage to right inferior frontal gyrus in humans. Nat Neurosci [Erratum (2003) 6:1329] 6:115-116.

Aron AR, Behrens TE, Smith S, Frank MJ, Poldrack RA (2007) Triangulating a cognitive control network using diffusion-weighted magnetic resonance imaging (MRI) and functional MRI. J Neurosci 27:3743-3752.

Band GP, van der Molen MW, Logan GD (2003) Horse-race model simulations of the stop-signal procedure. Acta Psychol (Amst) 112:105-142.

Brass M, Haggard P (2007) To do or not to do: the neural signature of self-control. J Neurosci 27:9141-9145.

Brass M, Derrfuss J, von Cramon DY (2005) The inhibition of imitative and overlearned responses: a functional double dissociation. Neuropsychologia 43:89-98.

Bunge SA, Dudukovic NM, Thomason ME, Vaidya CJ, Gabrieli JD (2002) Immature frontal lobe contributions to cognitive control in children: evidence from fMRI. Neuron 33:301-311.

Cai W, Leung HC (2009) Cortical activity during manual response inhibition guided by color and orientation cues. Brain Res 1261:20-28.

Cai W, Leung HC (2011a) Rule-guided executive control of response inhibition: functional topography of the inferior frontal cortex. PLoS One 6:e20840.

Cai W, Oldenkamp CL, Aron AR (2011b) A proactive mechanism for selective suppression of response tendencies. J Neurosci 31:5965-5969.

Chambers CD, Bellgrove MA, Stokes MG, Henderson TR, Garavan H, Robertson IH, Morris AP, Mattingley JB (2006) Executive "brake failure" following deactivation of human frontal lobe. J Cogn Neurosci 18:444-455.

Chao HH, Luo X, Chang JL, Li CS (2009) Activation of the presupplementary motor area but not inferior prefrontal cortex in association with short stop signal reaction time-an intra-subject analysis. BMC Neurosci 10:75.

Chikazoe J (2010) Localizing performance of go/no-go tasks to prefrontal cortical subregions. Curr Opin Psychiatry 23:267-272.

Chikazoe J, Konishi S, Asari T, Jimura K, Miyashita Y (2007) Activation of right inferior frontal gyrus during response inhibition across response modalities. J Cogn Neurosci 19:69-80.

Chikazoe J, Jimura K, Asari T, Yamashita K, Morimoto H, Hirose S, Miyashita Y, Konishi S (2009a) Functional dissociation in right inferior frontal cortex during performance of go/no-go task. Cereb Cortex 19:146-152.

Chikazoe J, Jimura K, Hirose S, Yamashita K, Miyashita Y, Konishi S (2009b) Preparation to inhibit a response complements response inhibition during performance of a stop-signal task. J Neurosci 29:15870-15877.

Coxon JP, Stinear CM, Byblow WD (2009) Stop and go: the neural basis of selective movement prevention. J Cogn Neurosci 21:1193-1203.

de Zubicaray GI, Andrew C, Zelaya FO, Williams SC, Dumanoir C (2000) Motor response suppression and the prepotent tendency to respond: a parametric study. Neuropsychologia 38:1280-1291.

Duann JR, Ide JS, Luo X, Li CS (2009) Functional connectivity delineates distinct roles of the inferior frontal cortex and presupplementary motor area in stop signal inhibition. J Neurosci 29:10171-10179.

Durston S, Thomas KM, Worden MS, Yang Y, Casey BJ (2002) The effect of preceding context on inhibition: an event-related fMRI study. Neuroimage 16:449-453.

Eagle DM, Baunez C, Hutcheson DM, Lehmann O, Shah AP, Robbins TW (2008) Stop-signal reaction-time task performance: role of prefrontal cortex and subthalamic nucleus. Cereb Cortex 18:178-188.

Feredoes E, Tononi G, Postle BR (2006) Direct evidence for a prefrontal contribution to the control of proactive interference in verbal working memory. Proc Natl Acad Sci U S A 103:19530-19534.

Forstmann BU, Jahfari S, Scholte HS, Wolfensteller U, van den Wildenberg WP, Ridderinkhof KR (2008) Function and structure of the right inferior frontal cortex predict individual differences in response inhibition: a model-based approach. J Neurosci 28:9790-9796.

Forstmann BU, Keuken MC, Jahfari S, Bazin PL, Neumann J, Schäfer A, Anwander A, Turner R (2012) Cortico-subthalamic white matter tract strength predicts interindividual efficacy in stopping a motor response. Neuroimage 60:370-375.

Garavan H, Ross TJ, Stein EA (1999) Right hemispheric dominance of in- 
hibitory control: an event-related functional MRI study. Proc Natl Acad Sci U S A 96:8301-8306.

Genovese CR, Lazar NA, Nichols T (2002) Thresholding of statistical maps in functional neuroimaging using the false discovery rate. Neuroimage 15:870-878.

Hampshire A, Chamberlain SR, Monti MM, Duncan J, Owen AM (2010) The role of the right inferior frontal gyrus: inhibition and attentional control. Neuroimage 50:1313-1319.

Hazeltine E, Bunge SA, Scanlon MD, Gabrieli JD (2003) Materialdependent and material-independent selection processes in the frontal and parietal lobes: an event-related fMRI investigation of response competition. Neuropsychologia 41:1208-1217.

Hester RL, Murphy K, Foxe JJ, Foxe DM, Javitt DC, Garavan H (2004) Predicting success: patterns of cortical activation and deactivation prior to response inhibition. J Cogn Neurosci 16:776-785.

Hirose S, Chikazoe J, Jimura K, Yamashita K, Miyashita Y, Konishi S (2009) Sub-centimeter scale functional organization in human inferior frontal gyrus. Neuroimage 47:442-450.

Hodgson T, Chamberlain M, Parris B, James M, Gutowski N, Husain M, Kennard C (2007) The role of the ventrolateral frontal cortex in inhibitory oculomotor control. Brain 130:1525-1537.

Horn NR, Dolan M, Elliott R, Deakin JF, Woodruff PW (2003) Response inhibition and impulsivity: an fMRI study. Neuropsychologia 41:1959-1966.

Jahfari S, Waldorp L, van den Wildenberg WP, Scholte HS, Ridderinkhof KR, Forstmann BU (2011) Effective connectivity reveals important roles for both the hyperdirect (fronto-subthalamic) and the indirect (frontostriatal-pallidal) fronto-basal ganglia pathways during response inhibition. J Neurosci 31:6891-6899.

Kelly AM, Hester R, Murphy K, Javitt DC, Foxe JJ, Garavan H (2004) Prefrontal-subcortical dissociations underlying inhibitory control revealed by event-related fMRI. Eur J Neurosci 19:3105-3112.

Kimura HM, Hirose S, Kunimatsu A, Chikazoe J, Jimura K, Watanabe T, Abe O, Ohtomo K, Miyashita Y, Konishi S (2010) Differential temporoparietal cortical networks that support relational and item-based recency judgments. Neuroimage 49:3474-3480.

Konishi S, Nakajima K, Uchida I, Sekihara K, Miyashita Y (1998) No-go dominant brain activity in human inferior prefrontal cortex revealed by functional magnetic resonance imaging. Eur J Neurosci 10:1209-1213.

Konishi S, Nakajima K, Uchida I, Kikyo H, Kameyama M, Miyashita Y (1999) Common inhibitory mechanism in human inferior prefrontal cortex revealed by event-related functional MRI. Brain 122:981-991.

Lenartowicz A, Verbruggen F, Logan GD, Poldrack RA (2011) Inhibitionrelated activation in the right inferior frontal gyrus in the absence of inhibitory cues. J Cogn Neurosci 23:3388-3399.

Leung HC, Cai W (2007) Common and differential ventrolateral prefrontal activity during inhibition of hand and eye movements. J Neurosci 27:9893-9900.

Li CS, Huang C, Constable RT, Sinha R (2006) Imaging response inhibition in a stop-signal task: neural correlates independent of signal monitoring and post-response processing. J Neurosci 26:186-192.

Li CS, Yan P, Sinha R, Lee TW (2008) Subcortical processes of motor response inhibition during a stop signal task. Neuroimage 41:1352-1363.

Liddle PF, Kiehl KA, Smith AM (2001) Event-related fMRI study of response inhibition. Hum Brain Mapp 12:100-109.

Logan GD, Cowan WB (1984) On the ability to inhibit thought and action: a theory of an act of control. Psychol Rev 91:295-327.

Maguire RP, Broerse A, de Jong BM, Cornelissen FW, Meiners LC, Leenders KL, den Boer JA (2003) Evidence of enhancement of spatial attention during inhibition of a visuo-motor response. Neuroimage 20:1339-1345.

Mander BA, Reid KJ, Baron KG, Tjoa T, Parrish TB, Paller KA, Gitelman DR, Zee PC (2010) EEG measures index neural and cognitive recovery from sleep deprivation. J Neurosci 30:2686-2693.
Matsubara M, Yamaguchi S, Xu J, Kobayashi S (2004) Neural correlates for the suppression of habitual behavior: a functional MRI study. J Cogn Neurosci 16:944-954.

Menon V, Adleman NE, White CD, Glover GH, Reiss AL (2001) Errorrelated brain activation during a $\mathrm{Go} / \mathrm{NoGo}$ response inhibition task. Hum Brain Mapp 12:131-143.

Milham MP, Erickson KI, Banich MT, Kramer AF, Webb A, Wszalek T, Cohen NJ (2002) Attentional control in the aging brain: insights from an fMRI study of the stroop task. Brain Cogn 49:277-296.

Milham MP, Banich MT, Barad V (2003) Competition for priority in processing increases prefrontal cortex's involvement in top-down control: an event-related fMRI study of the stroop task. Brain Res Cogn Brain Res $17: 212-222$

Morimoto HM, Hirose S, Chikazoe J, Jimura K, Asari T, Yamashita K, Miyashita Y, Konishi S (2008) On verbal/nonverbal modality dependence of left and right inferior prefrontal activation during performance of flanker interference task. J Cogn Neurosci 20:2006-2014.

Nakata H, Sakamoto K, Ferretti A, Gianni Perrucci M, Del Gratta C, Kakigi R, Luca Romani G (2008) Somato-motor inhibitory processing in humans: an event-related functional MRI study. Neuroimage 39:1858-1866.

Roberts GM, Garavan H (2010) Evidence of increased activation underlying cognitive control in ecstasy and cannabis users. Neuroimage 52:429-435.

Rubia K, Russell T, Overmeyer S, Brammer MJ, Bullmore ET, Sharma T, Simmons A, Williams SC, Giampietro V, Andrew CM, Taylor E (2001) Mapping motor inhibition: conjunctive brain activations across different versions of go/no-go and stop tasks. Neuroimage 13:250-261.

Rubia K, Lee F, Cleare AJ, Tunstall N, Fu CH, Brammer M, McGuire P (2005) Tryptophan depletion reduces right inferior prefrontal activation during response inhibition in fast, event-related fMRI. Psychopharmacology 179:791-803.

Severens E, Kühn S, Hartsuiker RJ, Brass M (2012) Functional mechanisms involved in the internal inhibition of taboo words. Soc Cogn Affect Neurosci 7:431-435.

Swick D, Ashley V, Turken AU (2008) Left inferior frontal gyrus is critical for response inhibition. BMC Neurosci 9:102.

Swick D, Ashley V, Turken U (2011) Are the correlates of stopping and not going identical? Quantitative meta-analysis of two response inhibition tasks. Neuroimage 56:1655-1665.

Talairach J, Tournoux P (1988) Co-planar stereotaxic atlas of the human brain. New York: Thieme Medical Publishers.

van Gaal S, Ridderinkhof KR, Scholte HS, Lamme VA (2010) Unconscious activation of the prefrontal no-go network. J Neurosci 30:4143-4150.

Verbruggen F, Logan GD (2008) Response inhibition in the stop-signal paradigm. Trends Cogn Sci 12:418-424.

Verbruggen F, Aron AR, Stevens MA, Chambers CD (2010) Theta burst stimulation dissociates attention and action updating in human inferior frontal cortex. Proc Natl Acad Sci U S A 107:13966-13971.

Vink M, Kahn RS, Raemaekers M, van den Heuvel M, Boersma M, Ramsey NF (2005) Function of striatum beyond inhibition and execution of motor responses. Hum Brain Mapp 25:336-344.

Worsley KJ, Friston KJ (1995) Analysis of fMRI time-series revisitedagain. Neuroimage 2:173-181.

Xue G, Aron AR, Poldrack RA (2008) Common neural substrates for inhibition of spoken and manual responses. Cereb Cortex 18:1923-1932.

Zandbelt BB, Vink M (2010) On the role of the striatum in response inhibition. PLoS One 5:e13848.

Zhang S, Li CSR (2012) Functional networks for cognitive control in a stop signal task: independent component analysis. Human Brain Mapp 33:89-104

Zheng D, Oka T, Bokura H, Yamaguchi S (2008) The key locus of common response inhibition network for no-go and stop signals. J Cogn Neurosci 20:1434-1442. 